\title{
Comportamiento en el uso de redes sociales en Centroamérica y el Caribe
}

Por Ernst \& Young El Salvador S.A de C.V

\section{$>$ Comportamiento en el uso de redes sociales en Centroamérica y el Caribe}

En Centroamérica finalmente ya se puede hablar de una sociedad digital, donde el uso de las redes sociales es cuestión de todos los días, ante este panorama las empresas deben estar preparadas para afrontar los retos que plantea tener clientes conectados e informados 24/7.

En la conferencia iLifebelt Trends Summit en junio de este año, fue presentado el Sexto Estudio Anual sobre las redes sociales Sociales en Centroamérica y el Caribe, este presenta un análisis basado en la combinación de diferentes fuentes de datos para tener un panorama claro de la situación del uso de redes sociales Sociales en la región.

Para comprender el estatus y desarrollo de esta, se presentarán las estadísticas más recientes de la

\section{Principales hallazgos}

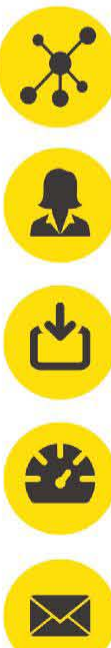

millennials acaparan uso de redes sociales en la región.

Crece el número de emprendedores entre los usuarios de redes sociales.

Internet continúa consolidándose los usuarios de redes sociales.

Velocidad de conexión, el principal problema reportado

Los hábitos más frecuentes son redes sociales y Correo Electrónico. industria digital de la mano de las principales tendencias que refleja el estudio.

La muestra del estudio fue de hombres y mujeres de 13 o más años internautas, usuarios de redes sociales en Centroamérica y República Dominicana, el nivel de confianza del estudio es del $95 \%(+-3.5 \%)$. Las respuestas se obtienen mediante una entrevista web asistida por ordenador (C.A.W.I. Computer Assisted Web Interview) por sus siglas en inglés, y se tomaron las respuestas de 785 individuos

Ante este panorama las empresas deben estar preparadas para afrontar los retos que plantea tener clientes conectados e informados 24/7.

Facebook y WhatsApp son las 2 redes sociales con más usuarios en la región.

WhatsApp la nueva Red Social favorita de los centroamericanos.

Nicaragua es el país con la mayor tasa de crecimiento en la cantidad de usuarios de Facebook en 2016

Centroamérica cuenta ya con 22 millones de usuarios de redes sociales.

Guatemala, el país con la mayor cantidad de usuarios de Facebook en centroamérica. 


\section{TEN D E N C I A S COMPORTAMIENTO EN EL USO DE REDES SOCIALES EN}

CENTROAMÉRICA Y EL CARIBE

\section{$>$ Tendencias}

\section{1 [Disminuye la brecha de género]}

En el estudio del año anterior los únicos dos países con mayor cantidad de usuarios femeninos eran Panamá y Honduras, sin embargo en esta nueva edición son las mujeres quienes casi lideran el uso de los medios digitales.
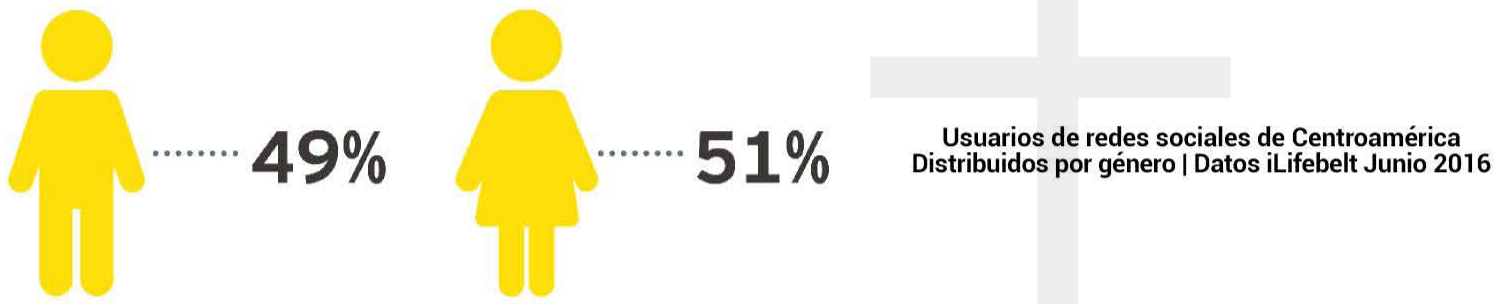

Honduras, Costa Rica, Panamá y República Dominicana son las naciones donde la mayor parte de los usuarios de redes sociales son mujeres. En el caso de Guatemala, El Salvador y Nicaragua la mayor parte de usuarios aún son varones.

Según los expertos una de las principales razones del aumento corresponde a la disminución de los costos de planes de datos y acceso a internet a través de dispositivos móviles, cuyo impacto se ve reflejado en todos los países, permitiendo que las mujeres que anteriormente no tenían la capacidad económica para acceder a Internet ahora puedan formar parte de la sociedad de la información. Una de las consideraciones de este fenómeno es tomar en cuenta las características socioeconómicas de la región.

\section{$2[$ Rango de edad]}

En la región el 55.5\% de los usuarios son Millennials seguidos por la generación Z, que incrementa constantemente la cantidad de usuarios, sin embargo cabe destacar que la generación post-millennial es muy diferente en cuanto al uso y código comunicacional que tienen Internet.

Esta generación representa a los principales adoptadores de redes como Snapchat, que empiezan a marcar las tendencias de hacia dónde va la comunicación digital. Son sumamente selectivos de la información que comparten y el grado de interacción con otros usuarios es determinante para ellos.

Por el contrario, los líderes de esta categoría son el grupo más amplio de consumidores a los que las marcas han tenido acceso en los últimos tiempos. Son jóvenes, están formados y están muy abiertos a gastar cantidades importantes en algo que les interesa.

\begin{tabular}{|l|l|l|}
\hline Rangos de Edad Usuarios Redes Sociales & 2015 & 2016 \\
\hline $\mathbf{1 2}$ a 20 años & $27 \%$ & $27.50 \%$ \\
\hline $\mathbf{2 1}$ a $\mathbf{3 0}$ años & $29.70 \%$ & $35.50 \%$ \\
\hline $\mathbf{3 1}$ a 40 años & $21.40 \%$ & $20 \%$ \\
\hline $\mathbf{4 1}$ a $\mathbf{5 0}$ años & $13.70 \%$ & $9.10 \%$ \\
\hline $\mathbf{5 1}$ a 60 años & $6.80 \%$ & $6.40 \%$ \\
\hline 61 o más & $1.40 \%$ & $1.50 \%$ \\
\hline Datos iLifebelt Junio 2016 & & \\
\hline
\end{tabular}

Las empresas se encuentran en proceso de comprender las necesidades y expectativas de los Millennials que representan el consumo actual, pero deben prepararse para ir creando posicionamiento con la nueva generación, que plantea retos completamente distintos. 
Uno de las tendencias sutiles que ha tomado fuerza conforme se posiciona la era digital en la región, es el aprovechamiento que le dan los usuarios a la concentración de público meta en las redes sociales, es por esto que incrementó el número de usuarios que se dedica al "trabajo por cuenta propia".

A diferencia de años anteriores, donde las dos principales actividades de los usuarios de redes sociales eran el trabajo por cuenta ajena seguido de la actividad académica (ser principalmente estudiante), hoy los usuarios se clasifican principalmente como empleados (44\%) y en segundo lugar como emprendedores (27\%). Queda relegado al tercer lugar la actividad académica 0 ser estudiante con un $23.2 \%$.

Ocupación de los Usuarios de Redes Sociales Datos iLifebelt Junio 2016

Fuente: Revista Mercados y Tendencias. ¿Cuál es el país más emprendedor de Centroamérica? Recopilado de: https://revistamyt.com/cual-es-el-pais-mas-emprendedor-de-centroamerica/

Esta tendencia digital coincide con otros indicadores del sector por ejemplo el mercado emprendedor latinoamericano es disputado por Colombia, Chile y Costa Rica, según reveló el Informe Condiciones Sistémicas para el Emprendimiento Dinámico (ICSED) 2015, países como Guatemala, El Salvador y República Dominicana se ubican al extremo opuesto del ranking, con variables inferiores a los 20 puntos en el índice de capital humano emprendedor.

En cuanto a factores que inciden sobre el espacio de oportunidades para emprender, Costa Rica, El Salvador, Guatemala y República Dominicana cuentan con un nivel medio en condiciones de la demanda; mientras Panamá se ubica en un nivel medio alto.

\section{4 [Problemas de los usuarios en redes sociales}

A los centroamericanos les gusta estar en redes sociales para enterarse de noticias y eventos (71.3\%), estar comunicación para saber que hacen los amigos (56.9\%), mantener contacto con otras personas $(43.9 \%)$, buscar entretenimiento (38\%) y llenar espacios de tiempo libre

(22.4\%), sin embargo una de las limitantes para el uso son los problemas de conexión, riesgos en seguridad y otras deficiencias que detallan los usuarios, en el siguiente gráfico de presentaron los resultados completos sobre los problemas al utilizar redes sociales.

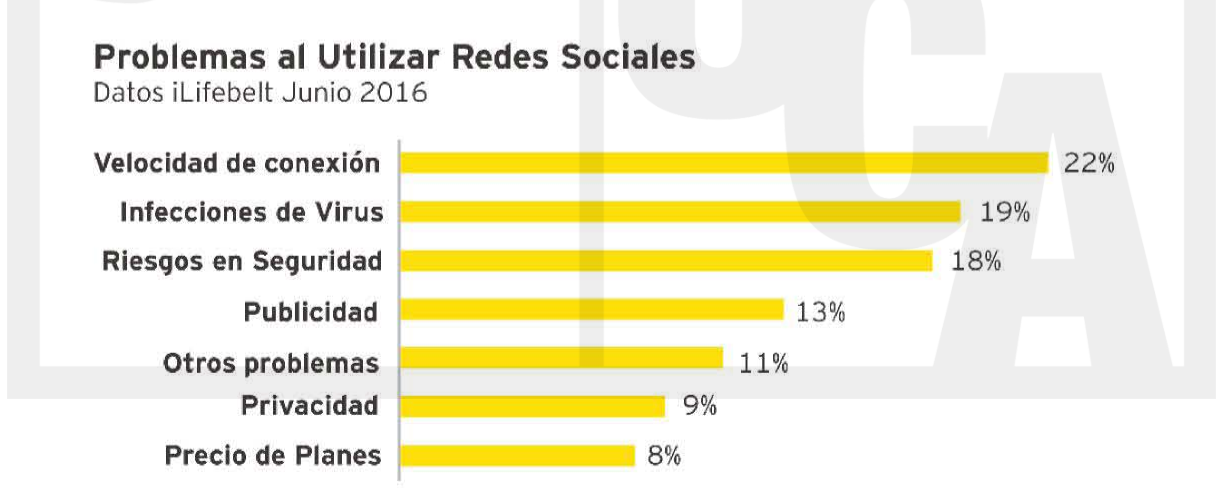

Fuente: Open Signal. Global State of the Mobile Network. Recopilado de: https://opensignal.com/reports/2016/08/global-state-of-the-mobile?network/ 


\section{TE N D E N C I A S COMPORTAMIENTO EN EL USO DE REDES SOCIALES EN}

La velocidad de conexión sigue siendo el principal problema de conectividad en la región. A pesar de los grandes avances que se han visto a lo largo de los últimos años, los usuarios siguen reportando la misma falla. Esta tabla muestra la velocidad total en cada país a través de sus redes de datos. Se define velocidad; como la conexión de datos móvil promedio que un usuario experimenta, basado en la velocidad y en la disponibilidad de las redes $3 G$ y $4 G$.

\begin{tabular}{|lll|}
\hline Países* & Posición en el Ranking & Megabites por Segundo \\
\hline Guatemala & 56 & $7.66 \mathrm{Mbps}$ \\
\hline Panamá & 66 & $6.15 \mathrm{Mbps}$ \\
\hline República Dominicana & 71 & $5.31 \mathrm{Mbps}$ \\
\hline El Salvador & 79 & $4.42 \mathrm{Mbps}$ \\
\hline Costa Rica & 94 & $2.55 \mathrm{Mbps}$ \\
\hline
\end{tabular}

*Nicaragua y Honduras no se encuentran en el ranking de OpenSignal

Es por lo anterior que la industria de telecomunicaciones requiere de una permanente inversión poder mantener la oferta de servicios de modo que mejore la calidad, pero el rezago en las tarifas y el vandalismo contra los equipos hacen que cada día sea más difícil la prestación de los servicios de Internet, telefonía móvil y television por suscripción en la región.

Finalmente, es el evidente incremento de la conciencia en relación a los riesgos que vienen acompañados de la red (phishing o suplantación de identidad, fraude informático, hacking antiético o lesivo, entre otros).

\section{$5[$ Redes sociales favoritas}

WhatsApp es la nueva Red Social favorita en Centroamérica y el Caribe. Es importante comprender que WhatsApp es un tipo de Red Social con sus propias características intrínsecas. Por su forma de interacción, se le puede clasificar como Red Celular Participativa (es decir, que se requiere del permiso del usuario para interactuar y son más aptas para la comunicación uno a uno por no contar con un "Timeline" nativo sino generado a través de los grupos).

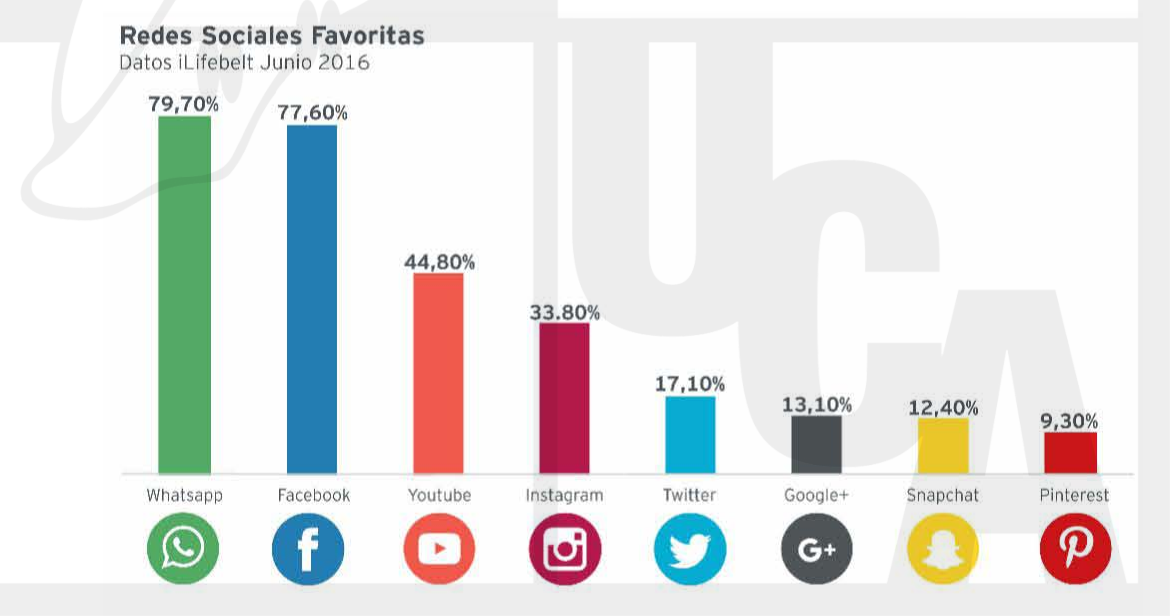

En el caso de Facebook, se trata también de una red tipo Celular, sin embargo, es Colectiva (nuevamente se requiere el permiso del usuario para interactuar pero es más apta para la comunicación a nivel grupal por poseer un "Timeline General".

El liderazgo de WhatsApp en preferencia se debe, entre otras cosas, a su simplicidad de uso, su rapidez en funcionamiento y el manejo más efectivo de la privacidad de los usuarios.

Fuente: GTM Tecno. Estudio Redes Sociales. Recopilado de: http://gtmtecno.com/2016/07/28/estudio-guatemaltecoredes-sociales/ 


\section{T E N D E N C I A S $\begin{aligned} & \text { COMPORTAMIENTO EN EL USO DE REDES SOCIALES EN } \\ & \text { CENTROAMERICA Y EL CARIBE }\end{aligned}$}

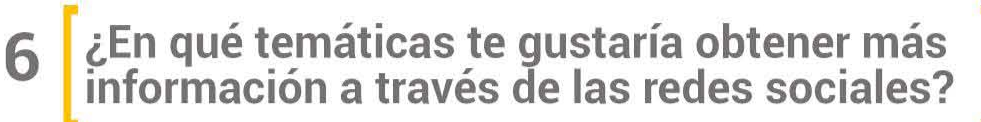

Estar informado, la razón más importante para seguir a una marca en Redes Sociales así lo confirma el $66.2 \%$ de los usuarios de Redes Sociales de la región que afirman que siguen a una marca por esta razón. , 14.3\% para conseguir ofertas, $12.6 \%$ porque se identifica con la marca, $6.5 \%$ para comprar productos o servicios y $0.4 \%$ para quejarse.

Sin embargo cuando los usuarios hablan de estar informados, se refieren a las siguientes temáticas que son las que tienen mayor porcentaje de interés entre los mismos.

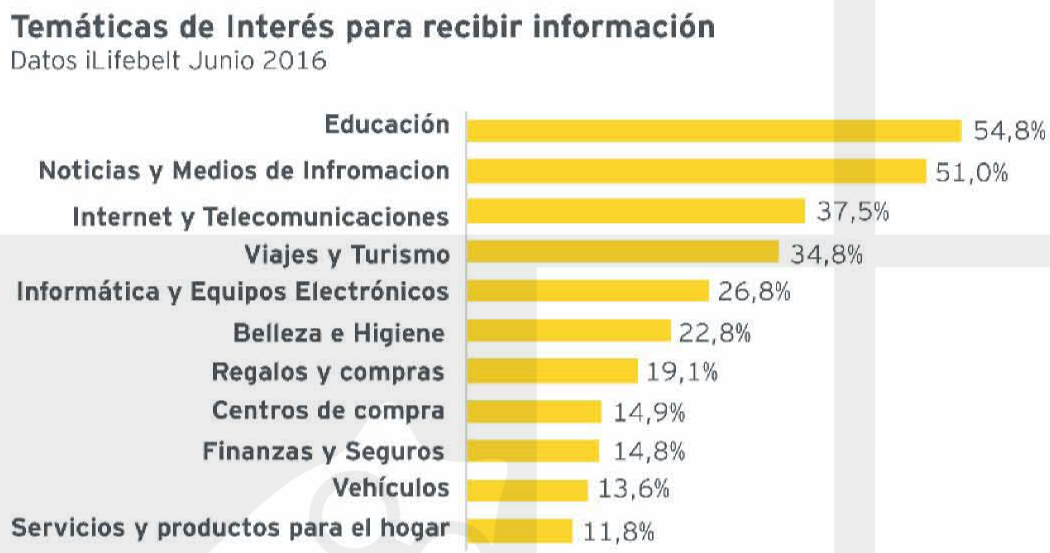

Las empresas deben tener en cuenta que el $82 \%$ de los usuarios de internet en Latinoamérica ha realizado compras online en el último año, porque lo que saber que temáticas compartir es vital para el posicionamiento de la marca y desarrollo de los clientes.

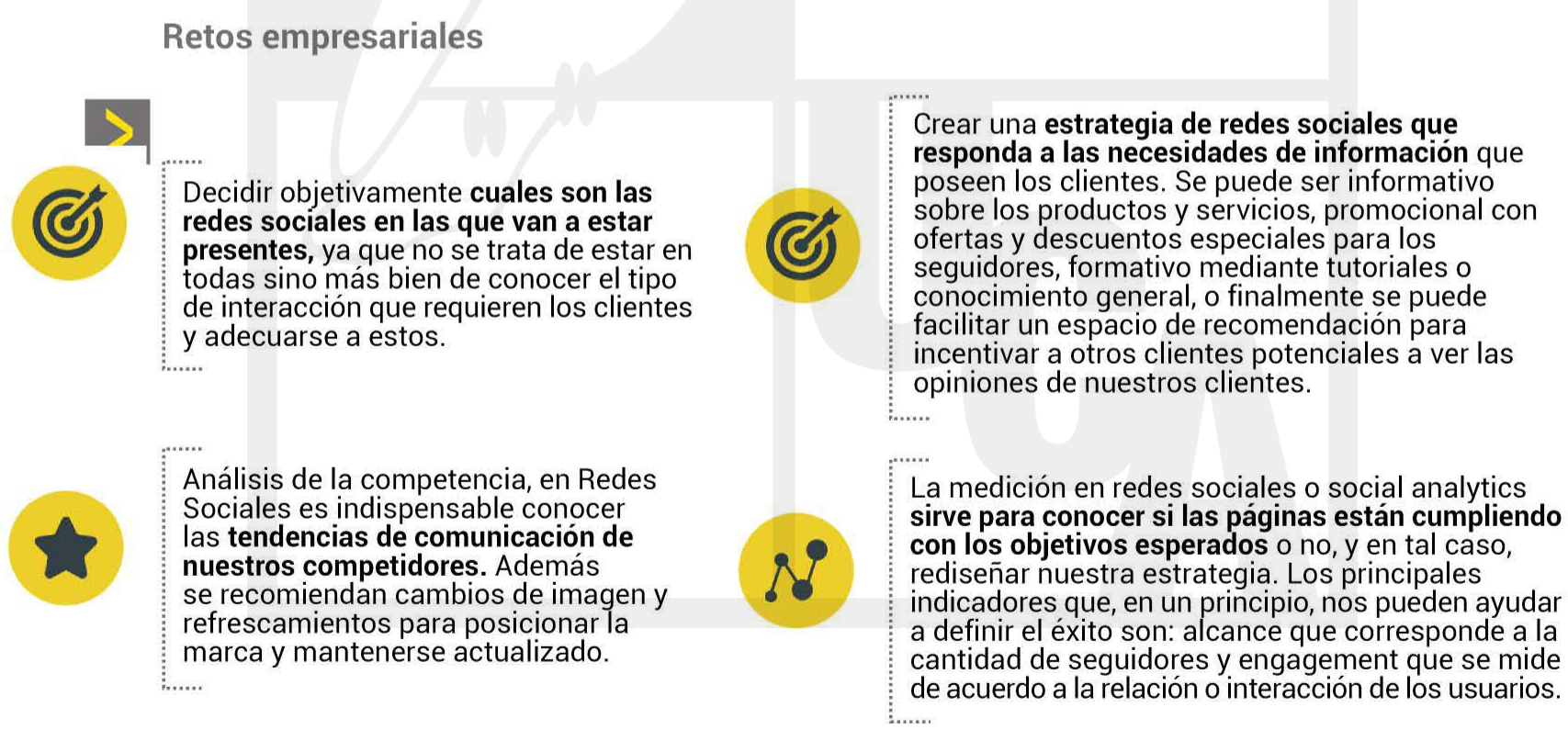

Fuente: Asociación Profesional de Empresarios de Instalaciones Eléctricas y Telecomunicaciones de Madrid. Recopilado de: http://apiem.org/ 


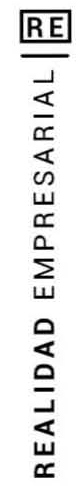

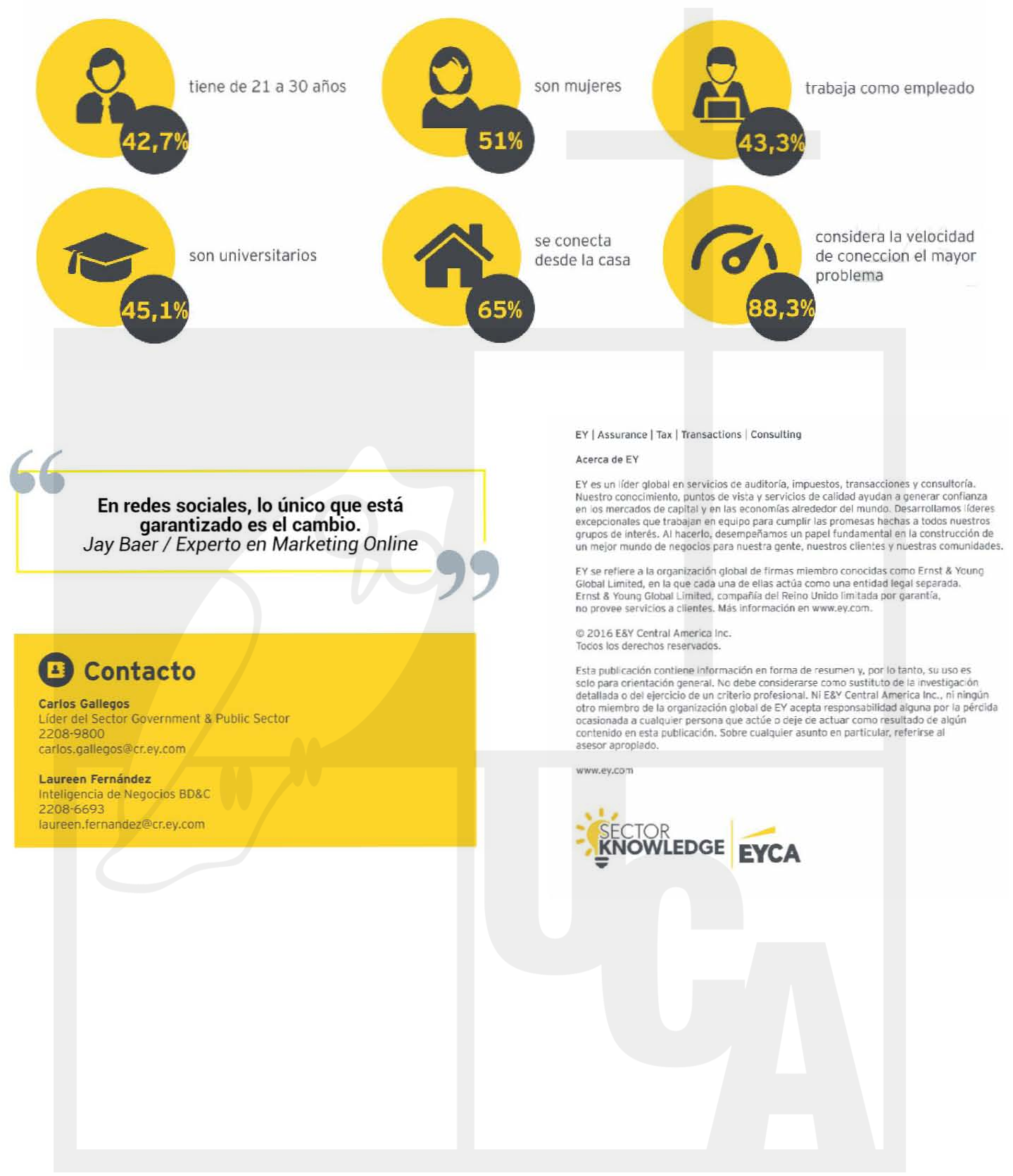

